\title{
Sonographic Diagnosis of Ascaris lumbricoides Infestation as a cause of Intestinal Obstruction
}

Sir,

A six-yr-old boy, presented with abdominal distension and vomiting with no passage of stool and gas for last four days. On clinical examination, patient was having distention of abdomen, with no guarding and rigidity. With the clinical diagnosis of intestinal obstruction, patient was rehydrated and then subjected to erect and supine radiographs of abdomen, which showed multiple air fluid levels of small bowel, suggestive of obstruction. Ultrasonography, which was performed with both convex array (3.5 MHz frequency) and linear array $(8.2 \mathrm{MHz}$ frequency) probes. The small gut loops were dilated, filled with food particles and fluid. On following the dilated loops on low frequency USG there were multiple longitudinal curvilinear echogenic strips with central anechoic linear area with no acoustic shadowing. On transverse section, these echogenic strips were rounded in shape with a peripheral echogenic line and central echogenic focus, with anechoic area in between. On high frequency transducer, intermingled multiple curvilinear echogenic strips each showing 3-line and 4-line appearances on longitudinal scan and target or bulls eye sign on transverse scan were seen, with few separate strips seen within dilated bowel loops (Figs. 1 and 2). The wall of the gut was normal in thickness. The diagnosis of round worms (Ascaris lumbricoides) causing intestinal obstruction was made on USG. The patient was operated upon and the diagnosis was confirmed. A large number of roundworms bunched together, were seen in the distal ileum, which were causing intestinal obstruction, were removed. Postoperative period and follow up were uneventful.

Large number of round worms can cause bowel obstruction, volvulus, intussusception, appendicitis, and

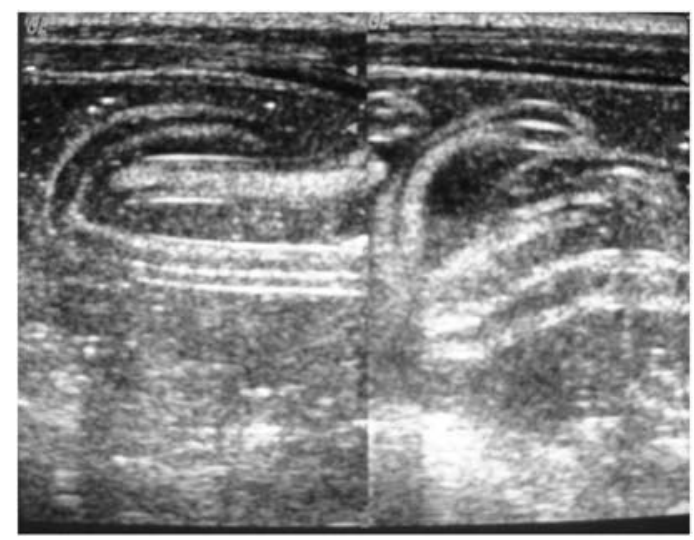

Fig. 1. Ultrasonography showing longitudinal three line and railway track appearance of worms in dilated intestine.

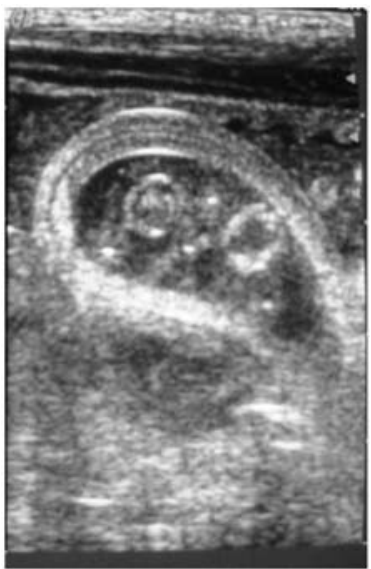

Fig. 2. USG showing both transverse (target sign) and longitudinal scans of worms.

even bowel perforation with penetration into the peritoneal cavity.

Ultrasonography of abdomen is a quick, safe, easily available, non-invasive and relatively inexpensive modality for suspected intestinal ascariasis. ${ }^{2}$ The various USG appearances of Ascaris lumbricoides have been described as: thick echogenic strip with central anechoic tube; multiple linear or curvilinear echogenic strips without acoustic shadowing; a "winding highway" or "parallel lines"; "railway track" sign; "3- line" or "4-line" sign on longitudinal scan and a "doughnut" or "target" sign and "bull's eye" appearances on transverse scan. The differential diagnosis of the round worm appearances on USG are nasogastric or jejunal feeding tube, surgical drainage tube, a ventriculoperitoneal shunt, or even a normal small bowel during peristalsis. ${ }^{2,3}$ However, when these USG features are seen within obstructed dilated fluid filled bowel loops, the diagnosis is easy to make.

Vinod Mehta, Sunder Goyal ${ }^{1}$, Sanjay Pandit ${ }^{1}$, Amit Mittal and Amit Aggarwal Departments of Radiodiagnosis and ${ }^{1}$ Surgery M.M. Institute of Medical Sciences and Research, Mullana, Ambala-133203, Haryana, India.

E-mail: amitmittalrod@yahoo.co.in [DOI-10.1007/s12098-010-0114-1]

\section{REFERENCES}

1. Barzilai M, Khamaysi N. Sonographic imaging of Ascaris lumbricoides. Harefuah 1996; 131: 247-248.

2. Peck RJ. Ultrsonography of intestinal ascaris. J Clin Ultrasound 1990; 18: 741-743.

3. Mahmood T, Mansoor N, Quraishy S, Ilyas M, Hussain S. Ultrasonographic appearance of Ascaris lumbricoides in the small bowel. J Ultrasound Med 2001; 20: 269-274. 